\title{
Schulterinstabilität im Sport: Diagnostik und Therapie
}

\author{
Martin Ophey
}

\begin{abstract}
Das Tennismatch mit unzähligen Aufschlägen, der geblockte Sprungwurf beim Handball, der Sturz auf den ausgestreckten Arm beim Skifahren, und schon ist es passiert: Das Schultergelenk (sub-)luxiert und wird instabil. Die Patienten berichten über Unsicherheitsgefühle bei bestimmten Bewegungen. Um die Schmerzursachen zu identifizieren, bieten sich klinische Muster und spezifische Tests an. Für die Therapie eignet sich das Kräftigen muskulärer Synergisten - von zentral nach peripher.
\end{abstract}

Verrenkungen des Schultergelenks sind mit einer Inzidenz von 14,7 pro 100000 Einwohner die häufigste Luxationsart und gehören zum Tagesgeschäft in den Notfallambulanzen [6,32]. Subluxationen sowie milde Instabilitäten kommen vermutlich noch viel häufiger vor, bleiben jedoch zumeist unerkannt. Dementsprechend liegen im Gegensatz zum Thema ,Luxation der Schulter - kaum Publikationen mit genauen Angaben zur Inzidenz von Subluxationen und milden Instabilitäten vor.

\section{Arten und Ursachen \\ der Schulterluxation}

\section{Erstluxationen}

Im zweiten sowie im sechsten Lebensjahrzehnt kommen Luxationen aufgrund sportlicher Aktivtäten bzw. wegen zunehmender Fallinzidenz gehäuft vor [28]. In 95 Prozent aller Fälle verursacht ein Trauma die Verrenkung des Schultergelenks [11].

Die weitaus selteneren nichttraumatischen Luxationen werden zumeist durch kleinere Verletzungen bei gleichzeitiger kapsuloligamentärer Insuffizenz und/oder koordinativer Probleme der stabilisierenden Muskulatur hervorgerufen.

APAS Die häufigste Luxations- bzw. Instabilitätsrichtung ist anterior. Demgemäß gebrauchen englischsprachige Autoren für die Schulterluxation häufig das Akronym ,APAS‘ (Acute Primary Anterior Shoulderdislocation).

\section{Rezidivluxationen}

Je jünger ein Patient bei einer Erstluxation ist, desto größer ist sein Risiko für eine rezidivierende oder habituelle Luxation - so denn nicht operativ eingegriffen wird [11]. Bei Patienten unter 20 Jahren liegt das Rezidivrisiko zwischen 72 und 95\%, im Alter zwischen 20 und 30 Jahren sinkt es auf 70 bis $80 \%$, bei den über 50 -jährigen beträgt es nur noch 14 bis $22 \%$.

OP-Indikation Über eine OP-Indikation entscheidet die patientenspezifische Analyse des Rezidivrisikos. Neben einem niedrigen Lebensalter sprechen Kontakt-, Wurfoder Schlagsportarten sowie beruflich bedingte Überkopftätigkeiten für eine chirurgische Intervention [4] ( $\bullet$ Abb. 1).

Weil das Rezidivrisiko nach einer Luxation und Reposition stark erhöht ist, ist der Erfolg einer konservativen Rehabilitation des Schultergelenks nicht gesichert, so dass im Zweifelsfall frühzeitig ein orthopädischer Chirurg konsultiert werden sollte.

\section{Anatomie und Biomechanik}

Unter anatomischen und biomechanischen Aspekten gelingt dem Schultergelenk ein Balanceakt zwischen Mobilität und Stabilität. Die Kontaktfläche zwischen Gelenkpfanne und Oberarmkopf beträgt nur 20\%. Im Zusammenspiel mit der weiträumigen Gelenkkapsel wird die Schulter so zum beweglichsten Gelenk des menschlichen Körpers.

Subsysteme Die Stabilität des Glenohumeralgelenks resultiert aus der Qualität der passiven, aktiven und neuralen Subsysteme [24, 25] (• Abb. 2). 


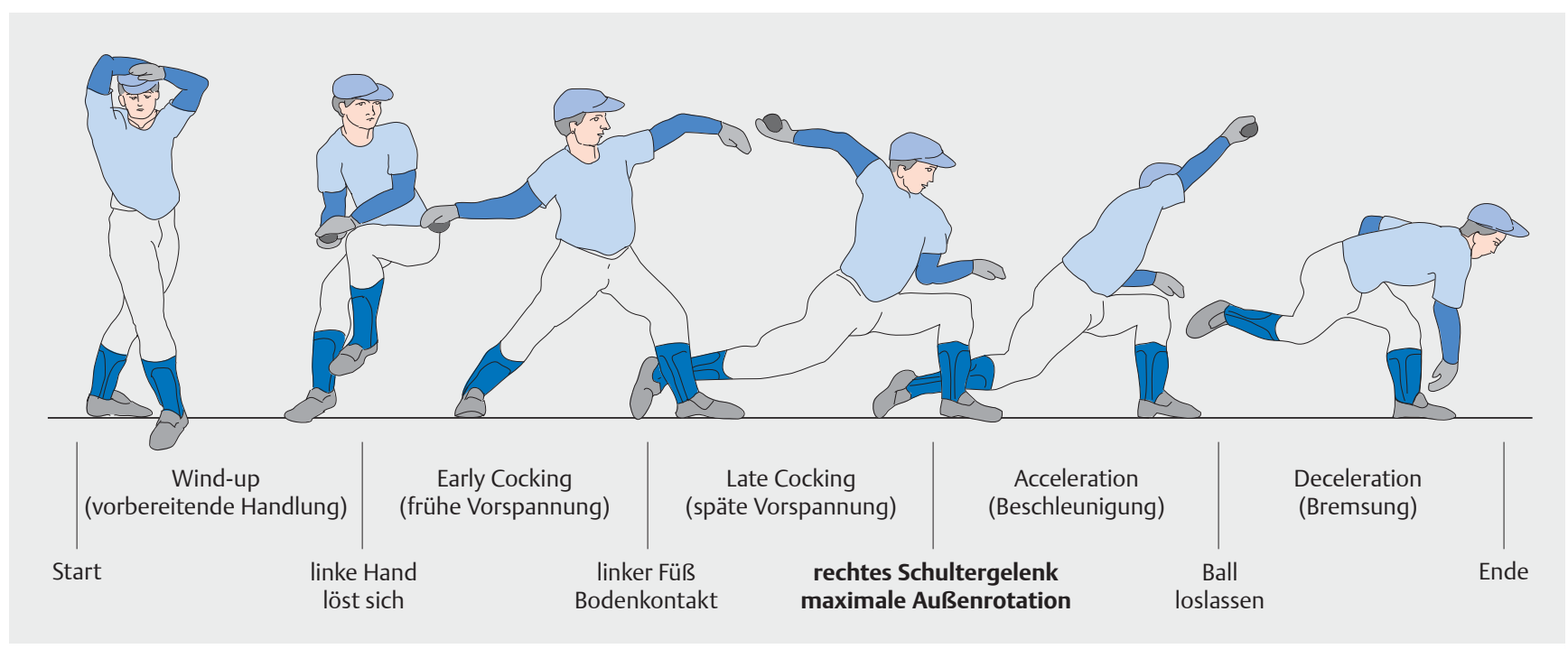

- Abb. 1 Risiko Wurfsportarten: Der Bewegungsablauf eines Baseball-Pitchers verdeutlicht die Belastung des Schultergelenks durch die große Abduktions-Außenrotation-Bewegungsamplitude in Verbindung mit einer hohen Beschleunigung. Nach dem Loslassen des Balls erfolgt das noch abruptere Abbremsen der Wurfbewegung.

Quelle: Thieme-Gruppe

\section{Passive Stabilisatoren}

Bei endgradiger Abduktion-Außenrotation spannt sich der ventrale Kapselbandapparat mit den interkapsulären Ligg. glenohumeralia. Während das geringgradige Abspreizen des Arms eher die obere und mittlere Kapsel sowie den superioren und medialen Teil der Ligg. glenohumeralia spannt, dehnen höhere Abduktionswinkel v. a. die untere Kapsel samt inferiorem Ligament.

Bei einer Schulterabduktion von mehr als $45^{\circ}$ verhindert v. a. das untere glenohumerale Band - mit seinem anterioren und posterioren Anteil umfasst es schlingenartig den Humeruskopf - eine exzessive Translation des Oberarmkopfes nach anterior, inferior und posterior [11]. Gleichzeitig verhindert der überstehende Rand des Labrum glenoidale ein übermäßiges Gleiten des Caput humeri nach anterior.

Die dorsale Schulterkapsel ist wesentlich dünner als der ventrale Kapselbandapparat. Sie sorgt für die posteriore Stabilität bspw. bei Stützaktivitäten mit $90^{\circ}$ Flexion [26]. Weil posteriore Instabilitäten bedeutend seltener imponieren, werden sie oft übersehen.

\section{Aktive Stabilisatoren}

Die aktive Stabilität der Schulter resultiert aus dem komplexen muskulären Zusammenspiel zwischen oberer und unterer Extremität sowie dem Rumpf. Infolgedessen ist dies gilt besonders für sportliche Leistungen - die effiziente Kraftübertragung von zentral nach peripher elementar [27].

\section{„Beginn nah am Zentrum, und arbeite dich nach außen vor!“}

Ein adäquates Reha-Programm zur Schulterrehabilitation berücksichtigt die kinematische Kette vom Körperkern hin zur oberen Extremität. Um die beteiligten Muskeln demgemäß in Gruppen zu klassifizieren, eignet sich die bereits im Jahre 1993 publizierte Einteilung nach Frank W. Jobe [16].

\section{Pivoters}

Das Zusammenspiel der zentralen Skapulafixatoren - sie werden als ,Pivoters` bezeichnet - garantiert eine gute Ausrichtung des Glenoids zum Humeruskopf. Bei perfektem Alignement sind das Kraft-Längen-Verhältnis der gelenkstabilisierenden Muskulatur sowie die Spannung des Kapselbandapparates optimal.

Dysfunktionelle Mobilitäts-, Koordinations- und Kraftdefizite der Skapulafixatoren sind sehr gut belegt $[7,17]$. Bei Sportlern imponiert häufig ein hypertoner M. pectoralis minor sowie eine schwache Pars ascendens des M. trapezius. Bei vorliegender Schulterinstabilität lässt sich der untere Trapezius u. a. sehr gut im geschlossenen System mittels Press-Ups im Sitzen aktivieren ( $\bullet$ Abb. $\mathbf{3}$ ). 


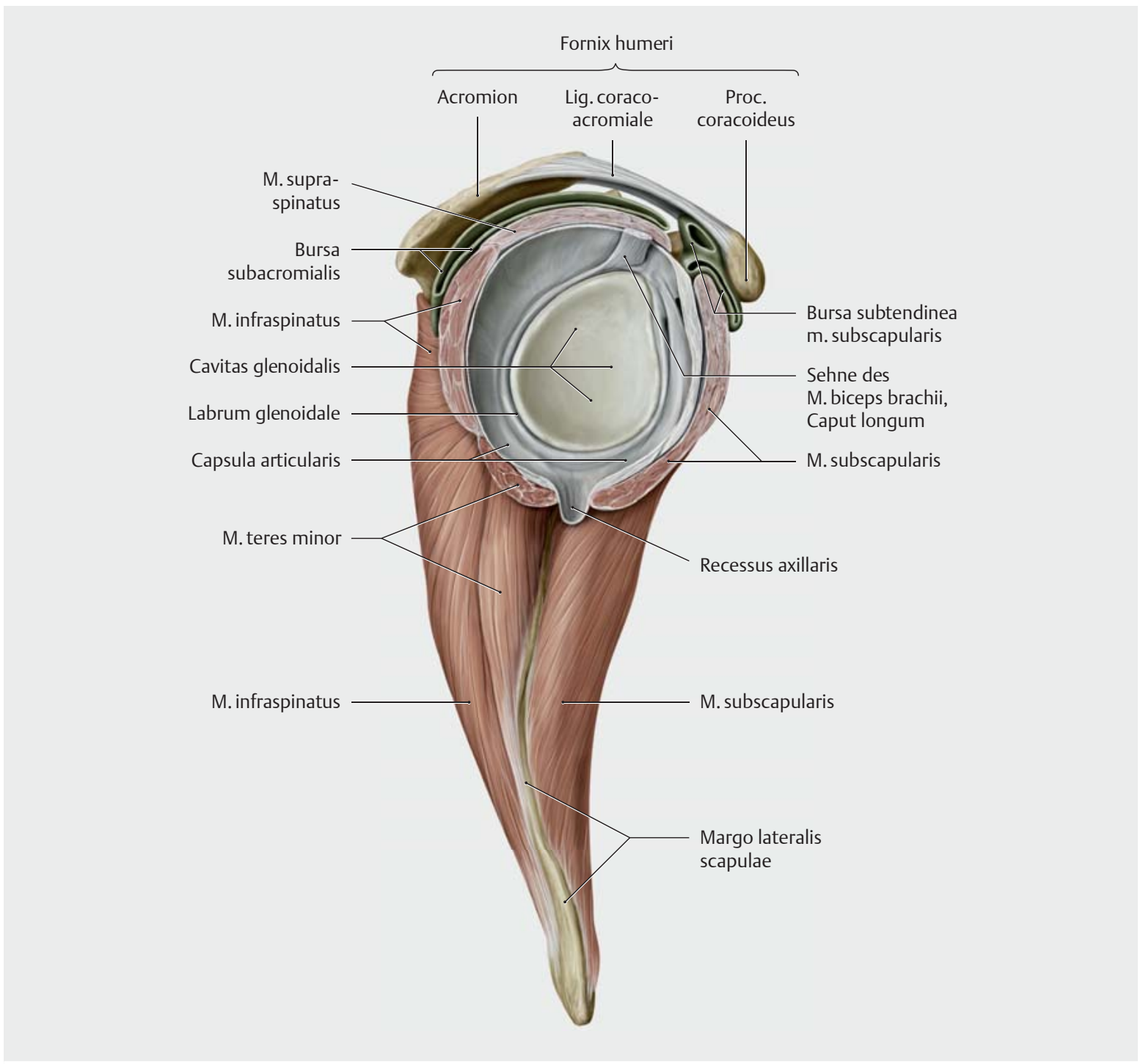

- Abb. 2 Cavitas glenoidalis mit durchtrennter Rotatorenmanschette. Kurz vor ihrem Ansatz am Humeruskopf strahlen die Muskeln der Rotatorenmanschette mit ihren Ansatzsehnen in die Gelenkkapsel ein und pressen den Humeruskopf manschettenartig in die Gelenkpfanne.

Quelle: Schünke M, Schulte E, Schumacher U. Prometheus. LernAtlas der Anatomie. Allgemeine Anatomie und Bewegungssystem. Illustrationen von K. Wesker. 4. Aufl. Stuttgart: Thieme; 2014.

\section{Protectors}

Als ,Protectors‘ zentrieren und kaudalisieren die Muskeln der Rotatorenmanschette sowie der Bizeps den Humeruskopf synergistisch in der Gelenkpfanne. Hierzu umschlingt das Kräftepaar aus den Mm. infraspinatus und teres minor gemeinsam mit dem M. subscapularis den Oberarmkopf. Bei simultaner Kontraktion zentrieren sie das Caput humeri in der Cavitas glenoidalis und spannen den Kapselbandapparat $[3,10]$. Für ein hocheffizientes und gleichzeitig sicheres Training der Außenrotatoren genügt ein Theraband ( $\triangleright$ Abb. 4).
Bizeps Bei Ermüdung des Caput longum des M. biceps brachii kann der Humeruskopf während einer Wurfbewegung verstärkt translatieren. Zudem verhindert der Bizeps die Kranialisation des Humeruskopfes und beugt so einem Impingement vor [14].

\section{Positioners}

Die Mm. supraspinatus und deltoideus spielen als ,Positioners' des Schultergelenks v. a. bei Flexion, Abduktion und Extension eine zentrale Rolle. Eine zu große Aktivität im Deltamuskel in Verbindung mit insuffizienten ,Protectors ‘ kann zu Impingement-Beschwerden führen oder diese aufrechterhalten. 


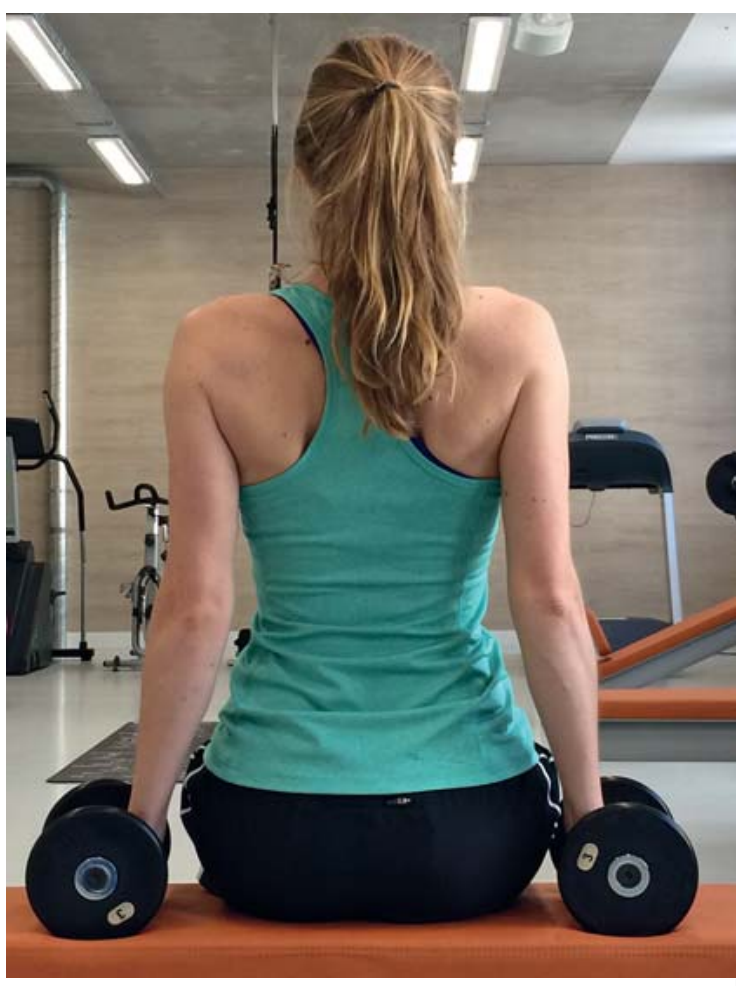

- Abb. 3 Sitting-Press-Up zum Training des unteren Trapezius bei insuffizienter Schulterblattfixation. Quelle: Martin Ophey.

\section{Propellors}

Die Mm. latissimus dorsi, pectoralis major und triceps brachii sorgen als ,Propellors` in erster Linie für Bewegungen des Arms. Aufgrund ihrer Leistungsfähigkeit können sie bei Fehlfunktionen anderer Muskelgruppen das Schultergelenk destabilisieren.

Plyometrie Patienten mit Schulterinstabilität, die schlag- oder wurfintensive Sportarten betreiben, arbeiten in der Endphase ihrer Rehabilitation plyometrisch ( $\bullet$ Abb. 5). Plyometrie bezeichnet eine Art von Schnellkrafttraining. Bei plyometrischen Übungen kommt der Dehnungs-Verkürzungs-Zyklus (DVZ) zum Tragen - also die Verlängerung des Muskels, gefolgt von einer plötzlichen, schnellen Verkürzung des Muskels. Der DVZ macht sich den Dehnreflex und die gespeicherte elastische Energie zunutze, um bei den Bewegungen mehr Kraft zu entwickeln.

\section{Preparators}

Die Muskulatur der unteren Extremität und des Rumpfes arbeiten als ,Preparators'. Sie finden sich nicht in der ursprünglichen Einteilung der aktiven Schulterstabilisatoren nach Jobe und wurden im Jahre 2007 in dessen Modell integriert $[1,16]$.

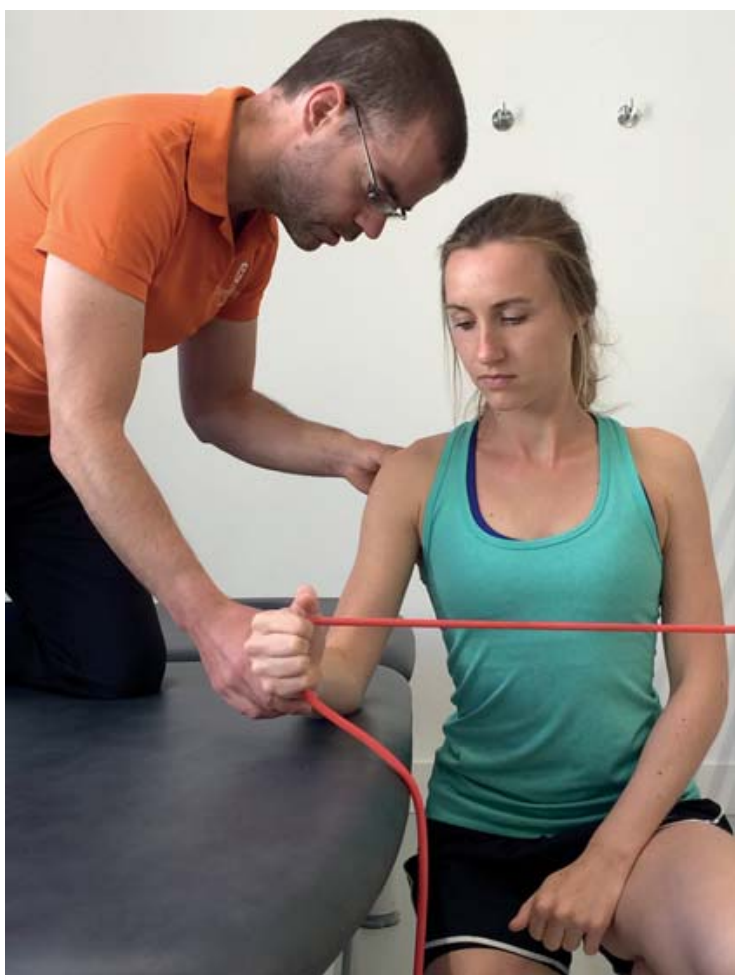

- Abb. 4 Theraband-Training der Außenrotatoren mit therapeutischer Unterstützung des optimalen Schulteralignements.

Quelle: Martin Ophey.

Die folgende Übersicht zeigt die Muskelgruppen der fünf Synergisten sowie geeignete Übungen für deren Aktivierung ( $\triangleright$ Tab. 1).

\section{Neurale Einflüsse}

Das Bindegewebe von Kapselbandapparat und Labrum glenoidale enthält außerordentlich viele Propriosensoren und freie Nervenendigungen [9,31]. Infolgedessen können diese passiven Strukturen ebenfalls zu Schmerzgeneratoren werden und reflektorisch die Protectors und Pivoters inhibieren [8].

\section{„Neurale Einflüsse können die Instabilitäts-Symptomatik triggern.“}

Aus diesem Umstand ergeben sich wesentliche Konsequenzen für die Dosierung der Trainingstherapie als zentraler Reha-Maßnahme für Patienten mit einer Schulterinstabilität. Länger bestehende Schmerzsyndrome des Schultergelenks können auf Rückenmarkebene sekundäre Hyperalgesien verursachen und zur zentralen Sensibilisierung führen [9]. 

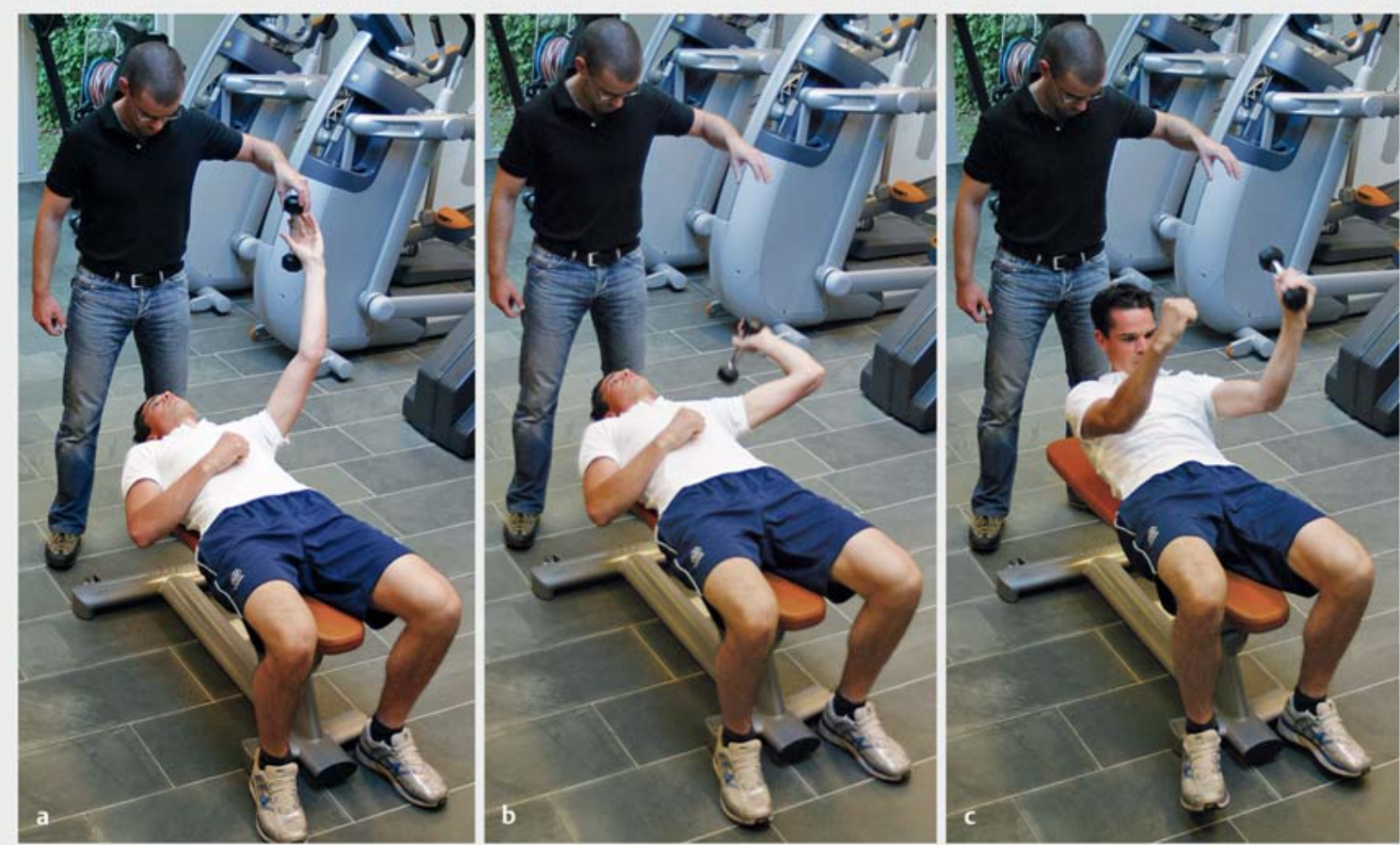

- Abb. 5 Transfersystem der oberen Extremität zur Vorbereitung auf das Werfen: schrittweises Ändern des Krafttrainings mit zunehmendem Gewicht und abnehmender Wiederholungsanzahl in ein Training mit abnehmendem Gewicht und zunehmender Geschwindigkeit.

Quelle: Martin Ophey.

\section{Diagnostik klinischer Instabilitätsmuster}

In der Physiotherapie ist die Anamnese das wesentlichste diagnostische Instrument. Um die aus dem Anamnesegespräch resultierenden Hypothesen zu bestätigen oder zu verwerfen, helfen gezielt ausgewählte körperliche Tests.

Ein adäquater diagnostischer Prozess hinterfragt zunächst das evtl. Vorliegen ernsthafter Pathologien als Kontraindikation für die physiotherapeutische Intervention und ergründet dann die Ursache(n) der Beschwerden sowie beitragende psychosoziale Faktoren. Im Anschluss wird nach effektiven und evidenzbasierten Einflussmöglichkeiten auf die Problematik des Patienten fahndet. Für die Beantwortung dieser Fragen sind klinische Muster hilfreich.

Leitsymptom Das Kardinalsymptom bei Patienten mit Instabilität sind (Sub-)Luxationen in der Vorgeschichte und/oder ein Unsicherheitsgefühl bei speziellen Haltungen und Bewegungen des Arms [30].
Sekundärer Schmerz Oft ist eines der beiden Hauptsymptome mit Schmerz verbunden, der sekundär als Folge der Instabilität entsteht. So kann eine Reizung der Rotatorenmanschette, das Caput longum des Bizeps oder die vordere/hintere Gelenkkapsel - in Form eines subakromialen bzw. dorsalen Impingements - massive Beschwerden verursachen [19]. Eben weil diese Schmerzen oft sekundär aus der primären Instabilität des Schultergelenks resultieren, ist es bei Patienten mit Schulterschmerzen unerlässlich, im Rahmen der Anamnese gezielt nach zurückliegenden (Sub-)Luxationen und Unsicherheitsgefühlen zu fragen.

Mustererkennung Ein Set aus Zeichen, Symptomen, dem Entstehungsmechanismus sowie dem Verlauf und den Patientenmerkmalen bildet ein klinisches Muster. Es ermöglicht Physiotherapeuten, gezielt den diagnostischen Prozess zu durchlaufen [13].

TUBS, AIOS, AMBRI und FI sind die akronymischen Abkürzungen für die häufigsten Instabilitätsmuster des Schultergelenks, die in zahlreichen Konzepten und Modellen Verwendung finden $[15,18,21]$ ( 
- Tab. 1 Die fünf P’s als aktive Stabilisatoren des Schultergelenks: Muskelgruppen und adäquate Übungen (modifiziert nach [16]).

\begin{tabular}{|c|c|c|}
\hline Synergisten & Muskeln & Übungen \\
\hline Pivoters & $\begin{array}{l}\text { - Skapulafixatoren: } \\
\text { - M. serratus anterior } \\
\text { - Mm. rhomboidei } \\
\text { - M. trapezius, Pars transversus } \\
\text { - M. trapezius, Pars ascendens }\end{array}$ & $\begin{array}{l}\text { - Press-Ups } \\
\text { - Elbow in the Pocket } \\
\text { - Wall Push-Ups } \\
\text { - Bent-over Barbell/Dumbbell Row } \\
\text { - Reversed Flys }\end{array}$ \\
\hline Protectors & $\begin{array}{l}\text { - Rotatorenmanschette: } \\
\text { - M. subscapularis } \\
\text { - M. supraspinatus } \\
\text { - M. infraspinatus } \\
\text { - M. teres minor } \\
\text { - M. biceps brachii }\end{array}$ & $\begin{array}{l}\text { - Belly Press } \\
\text { - Dynamic Hug } \\
\text { - Außenrotation } \\
\text { - Innenrotation } \\
\text { - Abduktion bis } 180^{\circ} \text { (Skapula-Ebene) }\end{array}$ \\
\hline Positioners & $\begin{array}{l}\text { - M. supraspinatus } \\
\text { - M. deltoideus }\end{array}$ & $\begin{array}{l}\text { - Front Raise } \\
\text { - Scaption Raise } \\
\text { - Lateral Raise } \\
\text { - Front Press } \\
\text { - Military Press } \\
\text { - Neck Press }\end{array}$ \\
\hline Propellors & $\begin{array}{l}\text { - M. pectoralis major } \\
\text { - M. latissimus dorsi } \\
\text { - M. triceps brachii }\end{array}$ & $\begin{array}{l}\text { - Bench Press } \\
\text { - Flys } \\
\text { - Peckdeck } \\
\text { - Push-Ups } \\
\text { - Pull-Over } \\
\text { - Lat Pull-Down } \\
\text { - Triceps Extension }\end{array}$ \\
\hline Preparators & $\begin{array}{l}\text { - Muskeln der unteren Extremität } \\
\text { - Muskeln des Rumpfes }\end{array}$ & $\begin{array}{l}\text { - Calf Raises } \\
\text { - Squat } \\
\text { - Squatlunge } \\
\text { - Lunge } \\
\text { - Barbell Rotation } \\
\text { - Back Extension } \\
\text { - Russian Twist }\end{array}$ \\
\hline
\end{tabular}

- Tab. 2 Abkürzungen der klinischen Muster instabiler Schultergelenke.

\begin{tabular}{|l|l|l|}
\hline Instabilitätsmuster & englisches Akronym & Bedeutung \\
\hline TUBS & - Traumatic Etiology & - traumatische Ursache \\
& - Unidirectional Instability & - unidirektionale Instabilität \\
\hline & - Bankart Lesion & - Bankart-Läsion \\
\hline & - Surgery & - Operation (Labrumrekonstruktion) \\
\hline AIOS & - Acquired & - erworbene \\
\hline & - Instability (of the) & - Instabilität (der) \\
\hline - Overstressed & - überlasteten \\
\hline AMBRI & - Shoulder & - Schulter \\
\hline & - Atraumatic Etiology & - atraumatische Ursache \\
& - Multidirectional Instability & - multidirektionale Instabilität \\
\hline - Bilateral & - bilateral \\
\hline FI & - Rehabilitation & - konservatives Management \\
& - Inferior Capsular Shift & - Kapselstraffung (Operation) \\
\hline
\end{tabular}




\section{TUBS}

Typische TUBS-Traumata sind blockierte Wurfbewegungen beim Handball oder ein Sturz auf den horizontal abduzierten Arm beim Skifahren oder Fußball. Die plötzliche große Krafteinwirkung translatiert den Humeruskopf nach anterior, wo der ventrokaudale Kapselbandapparat ihn bremsen muss.

Bankart-Verletzung Kleinere Traumata überdehnen lediglich den Kapselbandapparat. In der Regel vernarben die verletzten Strukturen im Rahmen der anschließenden Wundheilung gut. Größeren traumatischen Kräften halten die passiven Strukturen jedoch nicht stand; Kapsel-, Band- und Labrumrisse sind die Folge. Bei mehr als $80 \%$ der Patienten mit APAS-Luxation imponiert die klassische Bankart-Läsion mit Riss des ventrokaudalen Labrums. Die Ruptur reduziert die passive Stabilität erheblich, verschlechtert die Wundheilung und erhöht v. a. bei jungen Sportlern die Rezidivrate. Jüngere Luxationspatienten werden deshalb eher via operativer ,Bankart-Repair‘ stabilisiert.

Co-Läsionen Bei älteren Luxationspatienten können neben der Bankart-Läsion auch andere Begleitverletzungen auftreten wie bspw. Hill-Sachs-Läsionen, Glenoidoder Tuberculum-majus-Frakturen sowie Verletzungen des N. axillaris [29]. Die relativ oft auftretende Verletzung der Kapsel und des unteren Ligaments am Humerus bleibt meistens unerkannt. Diese so genannte ,HAGL-Läsion' (Humeral Avulsion of the Glenohumeral Ligament) kann an Rezidiv-Luxationen beteiligt sein [22].

\section{Merke \\ Begleitverletzungen \\ Angesichts der enormen Bandbreite von Begleit- verletzungen bei Schulterluxationen sollten bereits in der Frühphase Abweichungen von der normalen Wundheilung oder spontane Rezidiv-Luxationen erkannt und ggf. ein orthopädischer Chirurg kon- sultiert werden.}

\section{AIOS}

Beim AIOS-Muster entwickeln die Athleten die Instabilitäts- und Schmerzsymptomatik im Schultergelenk auf Grund permanenter Mikrotraumen langsam progredient [5]. Gerade bei wurfintensiven Sportarten findet man diese abgeschwächte TUBS-Form regelmäßig.

Anpassung des Gewebes Repetierende Überkopfbewegungen wie der Service beim Tennis und Volleyball oder das Werfen beim Hand-, Basket- und Baseball führen zu bindegewebigen Anpassungen. Entsprechend häufig beobachtet man bei Athleten dieser Disziplinen eine erhöhte Außenrotation und verminderte Innenrotation des Schultergelenks. Bis zu einem gewissen Grad handelt es sich um gezielte sportartspezifische Anpassungen, die sich nichtsdestotrotz zur Instabilitäts- und Schmerz-

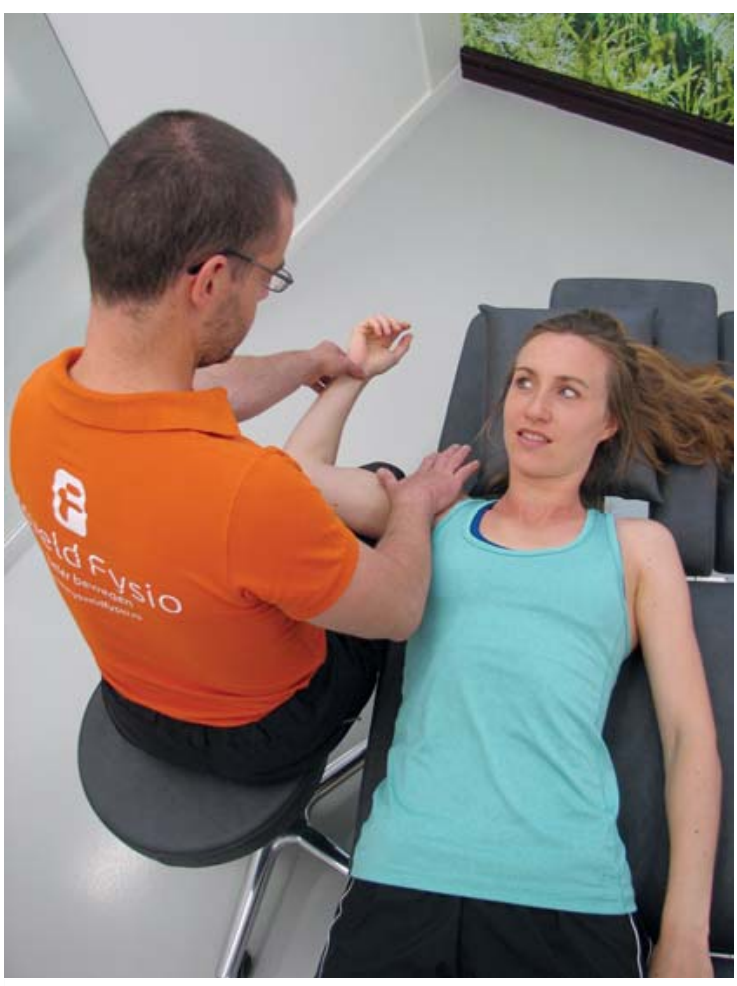

- Abb. 6 Apprehension-Test zur Identifikation einer milden AIOS-Instabilität.

Quelle: Martin Ophey.

symptomatik ausweiten können. Auch veränderte Wurftechniken, Trainingsformen, -umfänge sowie -intensitäten oder die fehlende Regeneration nach dem Sport können Symptome im Schultergelenk oder in gänzlich anderen Körperregionen wie bspw. der LWS oder Hüfte zur Folge haben.

\section{Untersuchung}

Mit dem Apprehension-, Relocation-, Release- oder dem Anterior-Drawer-Test lässt sich eine milde anteriore AIOS-Instabilität am besten feststellen [1, 12,23] ( $\bullet$ Abb. 6). Darüber hinaus sind auch unterhaltende Faktoren wie Bewegungseinschränkungen, Koordinationsund Kraftdefizite in der übrigen kinetischen Kette zu identifizieren.

Rehabilitation Zu Beginn der Behandlung muss der Athlet lernen, seine sportlichen Aktivitäten anzupassen. Fehlt dieser Lernprozess, erhält er seine Beschwerden aufrecht. Gerade dieser Knackpunkt macht die Therapie von Patienten mit einer AIOS-Instabilität so interessant. Vor der trainingsintensiven Rückgewinnung der sportlichen Leistungsfähigkeit sollte ein Therapeut evtl. vorliegende Bewegungseinschränkungen wie bspw. ein Innenund/oder Außenrotationsdefizit behandeln. 


\section{AMBRI}

Bei Patienten mit AMBRI-Muster existiert kein handfestes traumatisches Momentum, welches die Beschwerden erklärt. Ursache ist zumeist eine durch insuffizientes Bindegewebe bedingte generelle Laxität. Von dieser allgemein erhöhten Dehnfähigkeit des Bindegewebes sind verschiedene Gelenke betroffen.

Hypermobilität Die gesteigerte Dehnfähigkeit bzw. Hypermobilität imponiert in beiden Schultergelenken, d. h. auch im asymptomatischen, v. a. bei der Innen- und Außenrotation. Die passive Stabilität ist bei dieser ,multidirektionalen Instabilität‘ demzufolge nur mäßig, so dass aktive Stabilisatoren und neurale Systeme die Schulter kompensatorisch stabilisieren müssen.

AMBRI-Störungen entstehen auch ohne makroskopische Traumata bspw. durch erneute Wiederbelastungen. Zum Saisonstart nach der Sommerpause beobachtet man diese Instabilitätsproblematik häufig bei Wurfsportlern. Erneute Wiederbelastungen mit relativ rasch ansteigenden Trainingsumfängen und -intensitäten verursachen die Beschwerden.

\section{Untersuchung}

Mit dem Load-and-Shift-Test sowie dem Sulcus-Sign untersucht man das symptomatische und zum Vergleich auch das asymptomatische Schultergelenk ( Abb. 7, Abb. 8). Auch der Apprehension-Test kann bei Patienten mit AMBRI-Instabilität bilateral positiv sein ( $\bullet$ Abb. 6) $[12,15,23]$.

Rehabilitation Adäquate Reha-Maßnahmen verbessern die Stabilität und auch die Leistung. Notfalls kann die Kapsel später operativ gestrafft werden.

\section{$\mathrm{FI}$}

Die FI-Instabilität ist durch muskuläre Dysbalancen funktionell bedingt. In erster Linie verursachen muskuläre Kompensationsmechanismen die Beschwerden des FIMusters und erhalten sie aufrecht $[15,20]$.

Während beim klinischen TUBS- oder AMBRI-Muster das passiv stabilisierende System Probleme verursacht, die sich funktionell bei bestimmten Haltungen und Bewegungen äußern, inhibieren Patienten mit Fl-Muster kompensatorisch die Protectors, um mit den Propellors das Gelenk zu stabilisieren. Aufgrund seines Muskelquerschnitts und Verlaufes kann der kräftige M. pectoralis major eine glenohumerale Subluxation provozieren meist einhergehend mit einer schwachen Rumpfmuskulatur (Preparators) [15].

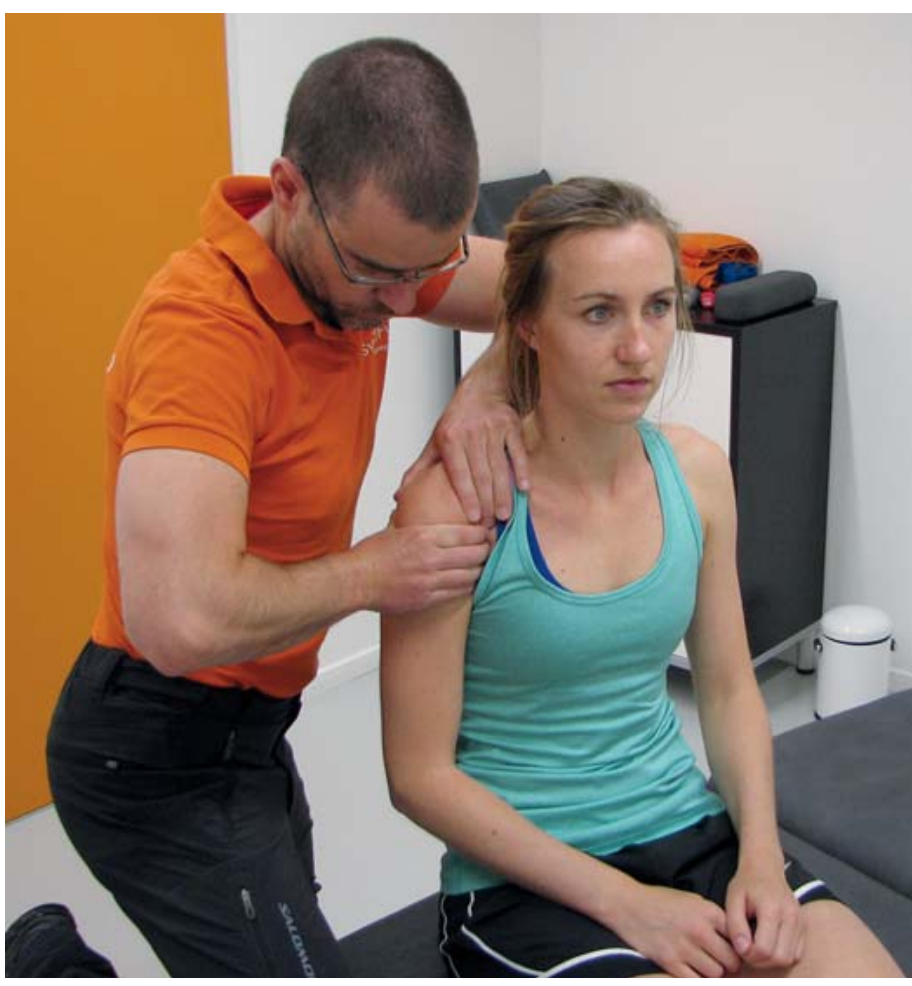

- Abb. 7 Load-and-Shift-Test zur Prüfung der anterioren und posterioren glenohumeralen Stabilität bei AMBRI-Patienten. Quelle: Martin Ophey.

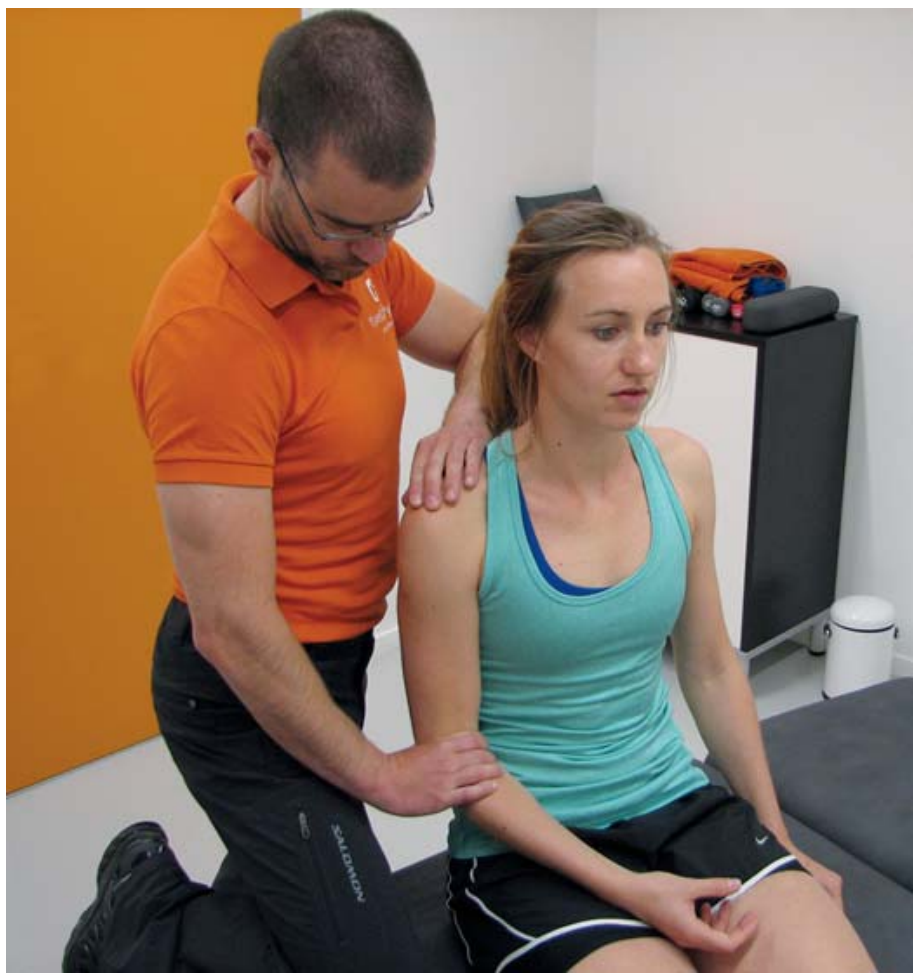

- Abb. 8 Via Sulcus-Sign kann der vergrößerte Gelenkspalt zwischen Humerus und Akromion bei bindegewebiger Laxität palpiert werden. Quelle: Martin Ophey. 


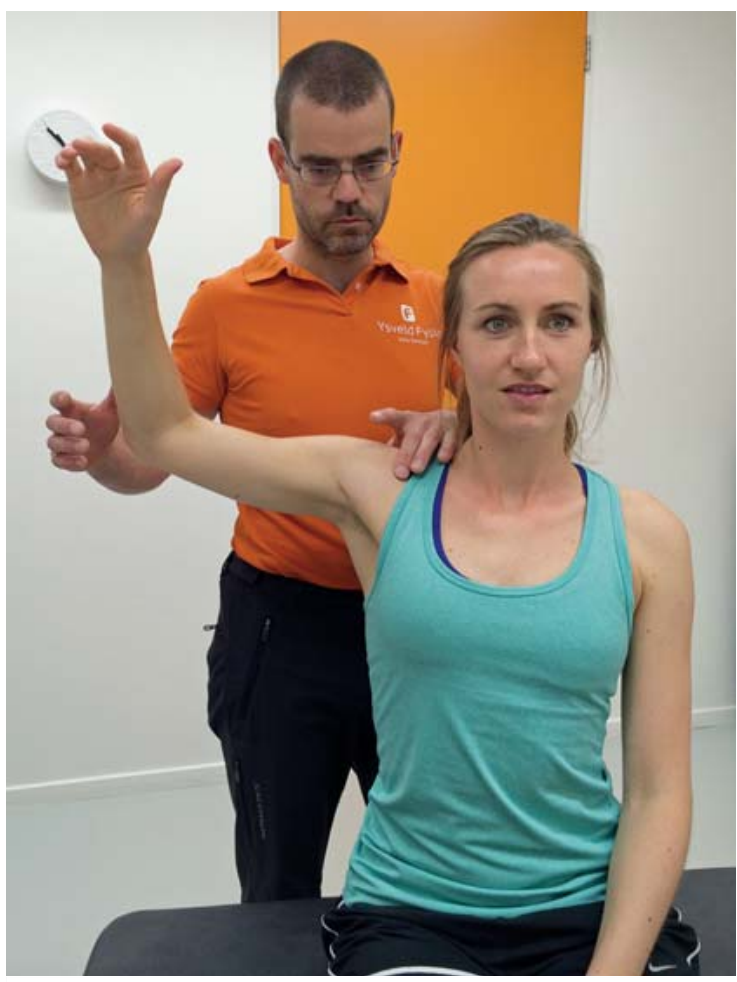

Abb. 9 Dynamic-Rotary-Stability-Test als motorischer Kontrolltest zur Feststellung einer funktionalen Schulterinstabilität.

Quelle: Martin Ophey.

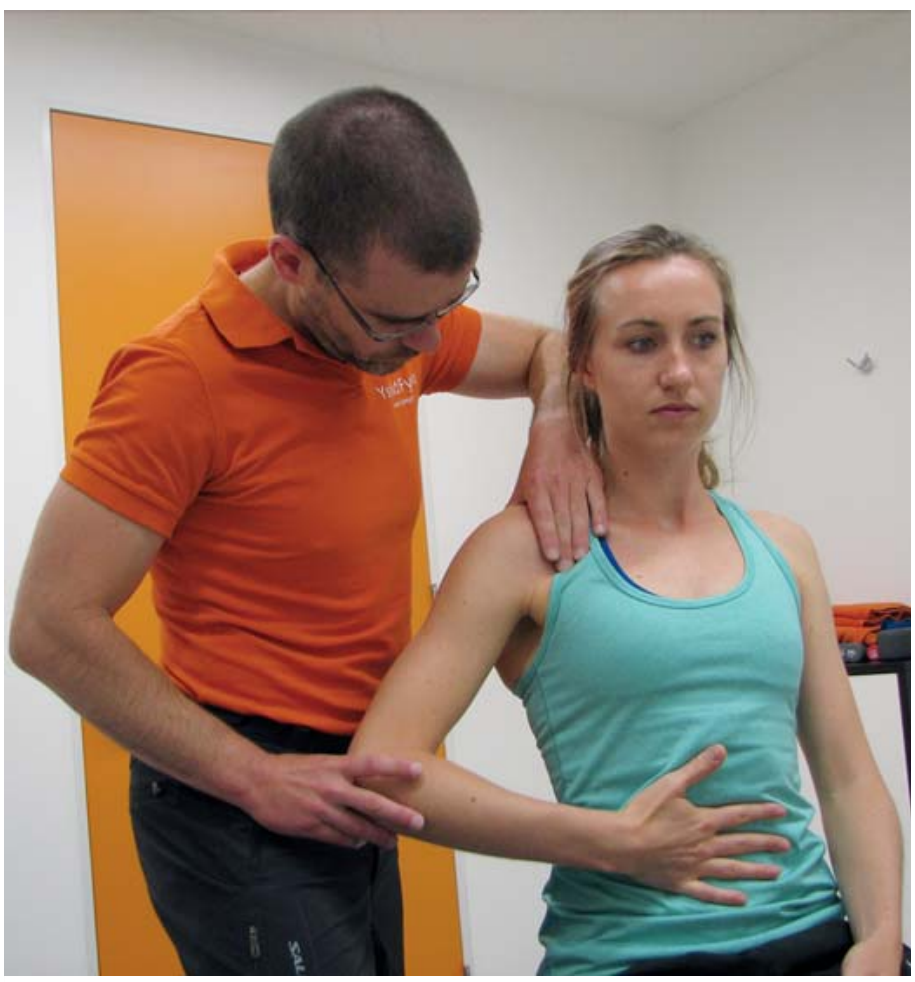

- Abb. 10 Belly-Press-Test zur Prüfung einer Hyperaktivität des M. pectoralis major bei Innenrotation des Arms. Quelle: Martin Ophey.

\section{Untersuchung}

Um maladaptive Kompensationsmechanismen zu entlarven, wird via Apprehension-Test zunächst eine strukturelle Instabilität ausgeschlossen. Motorische Kontrolltests wie der Dynamic-Rotary-Stability- sowie der Belly-PressTest sind zwar nicht ausreichend wissenschaftlich validiert, haben sich aber in der Praxis bewährt ( $\bullet$ Abb. 9, Abb. 10).

Beide Tests prüfen, ob der Humeruskopf übermäßig translatiert bzw. ob der M. pectoralis major zu stark aktiviert wird, und kontrollieren dabei die Bewegungsqualität. Beurteilung und Interpretation beruhen weitestgehend auf klinischer Erfahrung. Aus beiden Tests lassen sich sehr gut propriozeptive Übungen ableiten.

Rehabilitation Instabilitätspatienten mit klinischem FIMuster erhalten ein konsequentes konservatives RehaProgramm und trainieren in diesem Setting die komplette kinetische Kette.

\section{Fazit}

Neben früheren Luxationen sind Unsicherheitsgefühle bei bestimmten Bewegungen des Arms ein Kardinalsymptom für eine Instabilität des Schultergelenks. Demzufolge muss der Patient in der Anamnese gezielt nach solchen Unsicherheitsgefühlen befragt werden. Die vorgestellten klinischen Muster bieten eine profunde Orientierung für die Ursachenklärung - d. h. ob eine konkrete Verletzung, Mikrotraumata, insuffizientes Bindegewebe, muskuläre Dysbalancen im möglichen Zusammenspiel mit neuralen Faktoren ursächlich für die Problematik sind.

Häufig stagniert der Therapieverlauf bei Patienten mit einer Schulterinstabilität. Ist dies der Fall, muss nach Störfaktoren fahndet werden. Mittels der angeführten Untersuchungen lassen sich spezifisch lokale sowie allgemeine Faktoren, die den abweichenden Verlauf erklären, identifizieren. Als ,Leitplanken“ auf dem Weg zum stabilen Schultergelenk dienen die fünf P's ( $\bullet$ Tab. 1). Das Kräftigen dieser Synergisten erfolgt von zentral nach peripher bis der Patient sich sicher fühlt.

\section{Autorinnen/Autoren}

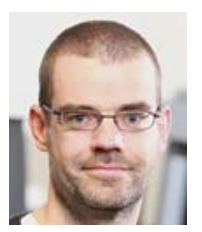

Martin Ophey arbeitet als Sportphysiotherapeut in einer Privatpraxis in Berg en Dal (NL) und doziert seit dem Jahre 2003 beim European Sportsphysiotherapy Education Network (ESP). Er hat sich auf die Behandlung von Sportlern mit Schulter-, Knie- und Sprunggelenksproblematik spezialisiert und ist Co-Autor des Thieme-Buches ,Sportphysiotherapie'. 


\section{Korrespondenzadresse}

\section{Martin Ophey}

IJsveldfysio

Zevenheuvelenweg 72

NL-6571 CK Berg en Dal

www.ysveldfysio.nl

martin_ophey@web.de

\section{Literatur}

[1] Bahk M, Keyurapan E, Tasaki A et al. Laxity testing of the shoulder: A review. Am J Sports Med. 2007; 35 (1): 131-144. doi:10.1177/0363546506294570

[2] Bant H, Haas H], Ophey M et al. Sportphysiotherapie. Stuttgart: Thieme; 2011

[3] Bant H, Ophey M. Hands-off: Training der SchultergelenkAußenrotatoren - Der richtige Dreh. physiopraxis 2012; 10 (10): 35-37. doi:10.1055/s-0032-1329730

[4] Boone JL, Arciero RA. First-time anterior shoulder dislocations: Has the standard changed? Br J Sports Med. 2010; 44 (5): 355-360. doi:10.1136/bjsm.2009.062596

[5] Castagna A, Garofalo R, Cesari E et al. Anterior and posterior internal impingement: An evidence-based review. Br J Sports Med. 2010; 44 (5): 382-388. doi:10.1136/bjsm.2009.059261

[6] CBO, Nederlandse Orthopedische Vereniging. Acute primaire schouderluxatie: Diagnostiek en Behandeling. Nijmegen: 2005. Im Internet: http://www.med-info.nl/Richtlijnen/ Bewegingsapparaat/Schouderluxatie.pdf; Stand: 28.05.2019

[7] Cools AM], Struyf F, De Mey K et al. Rehabilitation of scapular dyskinesis: From the office worker to the elite overhead athlete. Br J Sports Med. 2014; 48 (8): 692-697. doi:10.1136/ bjsports-2013-092148

[8] Cools AM, Witvrouw EE, Declercq GA et al. Scapular muscle recruitment patterns: trapezius muscle latency with and without impingement symptoms. Am J Sports Med 2003; 31 (4): 542-549. doi:10.1177/03635465030310041101

[9] Dean BJF, Gwilym SE, Carr AJ. Why does my shoulder hurt? A review of the neuroanatomical and biochemical basis of shoulder pain. Br J Sports Med 2013; 47 (17): 1095-1104. doi:10.1136/bjsports-2012-091492

[10] Gokeler A, Lehmann M. Tennis: Rehabilitation, Training and Tips. Sports Medicine and Arthroscopy Review 2001; 9: 105113

[11] Hayes K, Callanan M. Shoulder Instability: Management and rehabilitation. J Orthop Sports Phys Ther. 2002; 32 (10): $497-$ 509. doi:10.2519/jospt.2002.32.10.497

[12] Hegedus E], Goode AP, Cook CE et al. Which physical examination tests provide clinicians with the most value when examining the shoulder? Update of a systematic review with meta-analysis of individual tests. $\mathrm{Br}$ J Sports Med. 2012; 46 (14): 964-978. doi:10.1136/bjsports-2012-091066

[13] Higgs J, Jones M, Loftus $S$ et al. Clinical reasoning in the health professions. London: Butterworth-Heinemann Elsevier; 2008

[14] Itoi E, Newman SR, Kuechle DK et al. Dynamic anterior stabilizers of the shoulder with the arm in abduction. J Bone Joint Surg Br 1994; 76 (5): 834-836

[15] Jaggi A, Lambert S. Rehabilitation for shoulder instability. Br J Sports Med. 2010; 44 (5): 333-340. doi:10.1136/bjsm. 2009.059311
[16] Jobe FW, Pink M. Classification and treatment of shoulder dysfunction in the overhead athlete. J Orthop Sports Phys Ther. 1993; 18 (2): 427-432. doi:10.2519/jospt.1993. 18.2.427

[17] Kibler WB, Ludewig PM, McClure PW et al. Clinical implications of scapula dyskinesis in shoulder injury: The 2013 consensus statement from the 'scapula summit'.Br ] Sports Med. 2013; 47 (14): 877-885. doi:10.1136/bjsports-2013-092425

[18] Kuhn JE. Exercise in the treatment of rotator cuff impingement: A systematic review and a synthesized evidence-based rehabilitation protocol. J Shoulder Elbow Surg. 2009; 18 (1): 138-160. doi:10.1016/j.jse.2008.06.004

[19] Laudner K, Meister K, Noel B et al. Anterior glenohumeral laxity is associated with posterior shoulder tightness among professional baseball pitchers. Am J Sports Med. 2012; 40 (5): 1133-1137. doi:10.1177/0363546512437522

[20] Magarey ME, Jones MA. Specific evaluation of the function of force couples relevant for stabilization of the glenohumeral joint. Man Ther 2003; 8 (4): 247-253

[21] Magee D]. Orthopedic Physical Assessment. Philadelphia: Saunders; 2002

[22] Magee T. Prevalence of HAGL lesions and associated abnormalities on shoulder MR examination. Skeletal Radiol. 2014; 43 (3): 307-313. doi:10.1007/s00256-013-1778-1

[23] Moen MH, de Vos RJ, van Arkel ERA et al. De meest waardevolle klinische schoudertesten. Geneeskunde en Sport 2008; 41 (4): 6-17

[24] Myers J, Lephart S. Sensorimotor deficits contributing to glenohumeral instability. Clin Orthop Relat Res. 2002; (400): 98-104

[25] Panjabi MM. Clinical spinal instability and low back pain. J Electromyogr Kinesiol 2003; 13 (4): 371-379

[26] Provencher MT, LeClere LE, King S et al. Posterior instability of the shoulder. Am J Sports Med. 2011; 39 (4): 874-886. doi:10.1177/0363546510384232

[27] Putnam CA. Sequential motions of the body segments in striking and throwing skills: Descriptions and explanations. Journal of Biomechanics 1993; 26: 125-136

[28] Rowe CR. Prognosis in dislocations of the shoulder. J Bone Joint Surg 1956; 38-A (5): 957-977

[29] Sang JS, Yun YH, Kim DJ et al. Treatment of traumatic anterior shoulder dislocation in patients older than 60 years. Am J Sports Med. 2012; 40 (4): 822-827. doi:10.1177| 0363546511434522

[30] T'Jonck L. Functionele evaluatie en revalidatie bij patienten met glenohumerale instabiliteit en subacromiaal impingement. Leuven: KUL; 1998

[31] Vangsness CT, Ennis M, Taylor JG. Neural anatomy of the glenohumeral ligaments labrum and subacromial bursa. Arthroscopy 1995; 11 (2): 180-184

[32] Wheeler JH, Ryan JB, Arciero RA et al. Arthroscopic versus nonoperative treatment of acute shoulder dislocations in young athletes. Arthroscopy 1989; 5: 213-217

\section{Bibliografie}

DOI https://doi.org/10.1055/a-0888-1917

Der Schmerzpatient 2019; 2: 126-135

(c) Georg Thieme Verlag KG Stuttgart · New York ISSN 2512-6210 